\title{
Touch-ups, Rejuvenation, Re-dos and Revisions: Remote Communication and Cosmetic Surgery on the Rise
}

\author{
Roxanne H. Padley ${ }^{1}$ - Bruno Di Pace Br, $^{2,3}$
}

\begin{abstract}
The way in which communication takes place has undergone significant change over the last year leading to alterations regarding the way we perceive our own personal image. Indeed, the increase in remote video interaction seems to have led to an increase in the number of requests for teleconsultations with plastic surgeons in private practice (70\% in the UK and $64 \%$ in the USA), the so-called Zoom Boom. The reasons connected to this increase are still under investigation, but it is likely that patients' greater exposure to seeing their own image in online video communication for prolonged periods has highlighted what patients perceive as flaws and/or their self-image does not correspond to the same image that they see in the mirror. However, the motivations for seeking cosmetic surgery cannot be solely attributed to seeing ourselves online more frequently; the psychological impact of the pandemic (lower moods, isolation and greater concerns) may also be to blame. Therefore, the reasons patients hope to touch-up, rejuvenate, re-do or revise their "lockdown faces" need careful attention with a view to
\end{abstract}

Bruno Di Pace

dottbrunodipace@gmail.com

1 Department of Humanities, PhD School of Literary, Linguistic and Historical Studies, University of Salerno, Salerno, Italy

2 Department of Medicine, Surgery and Dentistry "Scuola Medica Salernitana", PhD School of Translational Medicine of Development and Active Aging, University of Salerno, Via Giovanni Paolo II 132, 84084 Fisciano, SA, Italy

3 Department of Plastic and Reconstructive Surgery, Addenbrooke's Hospital, Cambridge University Hospitals NHS Foundation Trust, Cambridge, United Kingdom

4 Anglia Ruskin School of Medicine, Anglia Ruskin University, Cambridge, United Kingdom understand the extent of which the online distortion of one's own body image may be the underlying cause.

Level of Evidence $V$ "This journal requires that authors assign a level of evidence to each article. For a full description of these Evidence-Based Medicine ratings, please refer to the Table of Contents or the online Instructions to Authors www.springer.com/00266."

Keywords Teleconsultations · Cosmetic surgery $\cdot$ Zoom boom - Lockdown face P Psychological impact . Rejuvenation

The way in which we communicate and how we go about our daily lives has undergone significant change over the last 12 months. The year anniversary since the first cases of SARS-COV-2 were detected in Europe now sees the vast majority of us interacting with others through some form of video conferencing be it for teaching purposes [1], research conferences or business meetings to name but a few. There has of course also been a rise in the use of telemedicine and teleconsultations [2-4] with a view to maintaining social distancing and reducing the spread of the virus and its variant mutations within hospital complexes.

Of particular note is that the increase in online video communication seems to have led to a specific increase in the requests for teleconsultations with plastic surgeons in the private sector. Indeed, the British Association of Aesthetic Plastic Surgeons (BAAPS) indicated a rise of $70 \%$ [5], a figure which is also reflected in the USA at 64\% [6]. This phenomenon has been coined as the "Zoom Boom" and gives rise to several considerations as to the reasons why more patients are seeking cosmetic procedures during a pandemic [5]. 
One of the most striking reasons is most likely to be the prolonged periods of time spent looking at yourself during a video conference which is far removed from the reality of everyday in person meetings. This new way of interacting has led us to become accustomed to seeing ourselves speaking and participating within our "new" working environments and has quite possibly led to a more critical view of our own self-perceptions and of our own bodyimage [7]. It may well be that the view we have of ourselves online does not correspond to the same view that we see of ourselves in the mirror, particularly as the amount of time looking in the mirror is most likely much reduced.

However, it is paramount that further considerations are made into the correlation between the incidence of an increase in video conferencing and a rise in patients who are seeking cosmetic surgery. Within the field of plastic surgery, with particular reference to reconstruction and breast cancer patients, a clear correlation has been established between levels of mood and a woman's perception of her own body image [8]. Indeed, the current pandemic has brought about the decision within most healthcare systems to defer all elective surgery, including breast reconstruction [9-11], which in itself is already signifying the psychological effects. Despite the fact that patients who undergo mastectomies and reconstructions in general [12-17] most certainly face greater challenges than patients who seek cosmetic surgery for aesthetic reasons, the psychological impact of self-isolation and the concerns connected to the pandemic cannot be ruled out as key motivators for requesting cosmetic surgery in this period.

Validated questionnaires which evaluate reconstructive plastic surgery procedures in terms of levels of satisfaction, body-image perception and levels of depression are already in wide use [7, 8, 18, 19]. Therefore, it would now be prudent to investigate the psychological impact of the pandemic on lower moods and potentially depression levels of patients and how this may or may not impact on a patient's distortion of their own body-image online leading to them seeking cosmetic surgery. To our knowledge, there are no current studies in this vein and the ways in which our own self-perception may now be distorted in online communication should now be investigated. The concept of a distorted self-perception of ourselves online is most likely connected to the fact that the way patients see their flaws are accentuated or indeed they believe that these "flaws" are now more noticeable to others more than before.

Another final consideration put forward is that patients have greater accessibility to surgeons through teleconsultations [20] and/or feel that smart working would allow for them to have a more comfortable healing time without the need to take sick leave. Financial considerations may also be pertinent as people are now spending less on other luxuries during lockdown.

Overall, the motivations which underpin a wish to touch-up, rejuvenate, re-do or revise your "lockdown face" need careful attention and the psychological impact of the pandemic on patients should not be overlooked in any way. Further studies in this sense should now be advanced in order to continue to guarantee the high standards and safety of patients who undergo cosmetic surgery.

\section{Declarations}

Conflict of interest The authors declare that they have no conflict of interest.

Human and Animal Rights This article does not contain any studies with human participants or animals performed by any of the authors.

Informed Consent For this type of study, informed consent is not required.

\section{References}

1. Padley RH, Di Pace B (2021) COVID-19 lockdown learning: the revolution of virtual teaching. J Plast Reconstr Aesthet Surg 74(2):407-447

2. Verdura V, Di Pace B, Cagiano L, Portincasa A (2021) Home wound management during the COVID-19 pandemic: the use of a novel adhesive retention suture. JAAD Case Rep 9:67-68

3. Sinha V, Malik M, Nugent N, Drake P, Cavale N (2020) The role of virtual consultations in plastic surgery during COVID-19 lockdown. Aesthetic Plast Surg. https://doi.org/10.1007/s00266020-01932-7

4. Gillman-Wells CC, Sankar TK, Vadodaria S (2021) COVID-19 reducing the risks: telemedicine is the new norm for surgical consultations and communications. Aesthetic Plast Surg 45(1):343-348

5. British Association of Aesthetic Plastic Surgeons. "Zoom Boom" - UK cosmetic surgeons see massive rise in demand for online consultations but urge caution. Available at: https://pressreleases. responsesource.com/news/99857/zoom-boom-uk-cosmeticsurgeons-see-massive-rise-in-demand/

6. American Society of Plastic Surgeons. ASPS Predicts New Industry Trends Amidst COVID-19 Reopenings. Available at: https://www.plasticsurgery.org/news/press-releases/americansociety-of-plastic-surgeons-predicts-new-industry-trends-amidstcovid19-reopenings

7. Hopwood P, Fletcher I, Lee A, Al Ghazal S (2001) A body image scale for use with cancer patients. Eur J Cancer 37(2):189-197

8. Di Pace B, Marotta M, Serlenga G et al (2020) Breast cancer and reconstruction: it changes inside and out. J Plast Reconstr Aesthet Surg 73(2):391-407

9. Di Pace B, Benson JR, Malata CM (2020) Breast reconstruction and the COVID-19 pandemic: a viewpoint. J Plast Reconstr Aesthet Surg 73(7):1357-1404

10. Di Pace B, Benson JR, Malata CM (2021) Breast reconstruction and the COVID-19 pandemic: adapting practice. J Plast Reconstr Aesthet Surg 74(3):644-710

11. Rocco N, Montagna G, Di Micco R et al (2021) The impact of the COVID-19 pandemic on surgical management of breast cancer: global trends and future perspectives. Oncologist 26(1):e66-e77 
12. Rubino C, Faenza M, Di Pace B, Campitiello N, Brongo S, Zingone G (2017) A new keystone flap "Plus" design: case series and analysis of follow-up. J Plast Reconstr Aesthet Surg 70(7):976-979

13. Di Pace B, Rubino C (2021) The modified keystone flap in periarticular or large superficial tumor resection surgery. J Surg Oncol 123(4):1164-1165

14. Di Pace B, Verdura V, Concilio M et al (2019) A rare case of squamous cell carcinoma of a proliferating trichilemmal tumour. Ann Ital Chir 88:263-267

15. Verdura V, Di Pace B, Concilio M et al (2019) A new case of radiation-induced breast angiosarcoma. Int J Surg Case Rep 60:152-155

16. Ngaage LM, Oni G, Di Pace B et al (2018) The effect of CT angiography and venous couplers on surgery duration in microvascular breast reconstruction: a single operator's experience. Gland Surg 7(5):440-448
17. Guastafierro A, Verdura V, Di Pace B, Faenza M, Rubino C (2019) The influence of breast cancer on the distribution of cherry angiomas on the anterior thoracic wall: a case series study. Dermatology 235(1):65-70

18. Pusic AL, Klassen AF, Scott AM, Klok JA, Cordeiro PG, Cano SJ (2009) Development of a new patient-reported outcome measure for breast surgery: the BREAST-Q. Plast Reconstr Surg 124(2):345-353

19. Hamilton M (1967) Development of a rating scale for primary depressive illness. Br J Soc Clin Psychol 6:278-296

20. Padley RH, Di Pace B (2021) Medical advances in telehealth and innovative communication: new directions. Gazz Med Ital - Arch Sci Med. https://doi.org/10.23736/S0393-3660.21.04606-4

Publisher's Note Springer Nature remains neutral with regard to jurisdictional claims in published maps and institutional affiliations. 